Bull. Mater. Sci., Vol. 36, No. 6, November 2013, pp. 973-976. (c) Indian Academy of Sciences.

\title{
$X$-ray determination of crystallite size and effect of lattice strain on Debye-Waller factors of platinum nano powders
}

\author{
E PURUSHOTHAM* and N GOPI KRISHNA \\ Department of Physics, Kakatiya University, Warangal 506 009, India
}

MS received 7 March 2012; revised 11 April 2012

\begin{abstract}
In the present study, nano platinum particles were produced by ball milling process. The lattice strains in platinum $(\mathrm{Pt})$ powders produced by milling have been analysed by $\mathrm{X}$-ray powder diffraction. The lattice strain $(\varepsilon)$ and Debye-Waller factor $(B)$ are determined from the half-widths and integrated intensities of the Bragg reflections. In Pt, the Debye-Waller factor is found to increase with lattice strain. From the correlation between the strain and effective Debye-Waller factor, the Debye-Waller factors for zero strain have been estimated for Pt. The variation of energy of vacancy formation as a function of lattice strain has been studied.
\end{abstract}

Keywords. X-ray diffraction; lattice strain; crystallite size; Debye-Waller factor; vacancy formation energy.

\section{Introduction}

Nanoparticles of size $<100 \mathrm{~nm}$ in diameter exhibit enhanced size-dependent properties compared to ultra-fine or larger particles of the same material. An accurate estimation of grain size/crystallite size becomes essential when such materials are produced with their crystallite size of the order of $<100 \mathrm{~nm}$. Though TEM is one of the powerful techniques for crystallite size measurement, it has certain limitations. Since TEM images represent only a local region, many samples and images are required to provide an average information for the entire sample. Not only this, TEM sample preparation method is an involved and time consuming one. XRD technique is free from these limitations. X-ray diffraction is, on the other hand, a simple and an easier approach for determination of crystallite size of powder samples.

The Debye-Waller factor is an important lattice dynamical property. There is considerable X-ray work on the DebyeWaller factors of Pt (Alexopoulos et al 1965). But it is interesting to study the effect of lattice strains on the DebyeWaller factors of this metal. Inagaki et al (1971, 1973) showed that in several non-metallic powders, the strains produced during grinding have a significant effect on the DebyeWaller factors measured from X-ray diffraction intensities. Sirdeshmukh et al (1993) observed the effect of lattice strains on the Debye-Waller factors in semiconductor powder materials. Gopi Krishna and Sirdeshmukh (1993) studied the effect of lattice strains on the Debye-Waller factor of ytterbium metal. In the present investigation, results of a systematic study of the effect of lattice strains on the Debye-Waller factors and crystallite size of face centred cubic Pt metal is reported. These results are being reported for the first time.

\footnotetext{
*Author for correspondence (psm45456@gmail.com)
}

\section{Experimental}

The powder samples were obtained by gently filing highly pure Pt metal ingots with a jeweller's file. A part of this powder was used to prepare the initial sample. The remaining powder was subjected to milling in a ball mill for 5, 10, 15, 20 and $25 \mathrm{~h}$ to produce strains. X-ray diffractograms were recorded with the initial sample and with samples prepared after each spell of milling. The diffractograms were obtained with a Philips CWU 3710 X-ray powder diffractometer in the $2 \theta$ range $20-120^{\circ}$ using filtered $\mathrm{CuK} \alpha$ at a goniometer speed of $0.5 \% \mathrm{~min}$ and a chart speed of $20 \mathrm{~mm} / \mathrm{min}$. All measurements were made at room temperature. The observed integrated intensities were corrected for thermal diffuse scattering using the method of Chipman and Paskin (1959).

\section{Analysis of data}

For the relative intensity method, expression for the observed intensities, $I_{0}$, is given by

$$
I_{0}=C L_{\mathrm{p}} J F_{\mathrm{T}}^{2},
$$

where $L_{\mathrm{p}}$ is the Lorentz-polarization factor, $J$ the multiplicity factor, $F_{\mathrm{T}}$ the structure factor and $C$ is a constant. For a flat powder specimen, the absorption correction is independent of the angle $\theta$ (Klug and Alexander 1974) and, hence, is lumped with the constant. The structure factor, $F_{\mathrm{T}}$, in terms of the structure factor, $F$, for the static lattice is given by

$$
F_{\mathrm{T}}=\mathrm{Fe}^{-\mathrm{M}} .
$$

We may also write (1) as

$$
I_{0}=I_{\mathrm{c}} e^{-2 B\left(\frac{\sin \theta}{\lambda}\right)^{2}},
$$


where $I_{\mathrm{c}}$ is the intensity corresponding to the static lattice and is given by

$$
I_{\mathrm{c}}=L_{\mathrm{p}} J F^{2} .
$$

For crystals with $f c c$ structure, structure factor, $F$, is given by

$$
F=4 f
$$

$f$ being the atomic scattering factor. Values of the atomic scattering factor were taken from Cromer and Waber (1965) and International Tables for X-ray Crystallography (1968), and have been corrected for dispersion (Cromer and Liberman 1970). The choice of the atomic scattering factors was guided by Cromer and Waber (1965) recommendation, who suggested that the atomic scattering factors given in the International Tables for X-ray Crystallography (1968) which are computed from the Hartree-Fock wave functions are the best for the lighter atoms or ions, in the periodic table up to $\mathrm{Rb}^{+1}$. For the heavier atoms or ions, Cromer recommends the use of Dirac-slater relativistic scattering factors given by Cromer and Waber (1965).

From (3), it can be seen that $\log \left(I_{0} / I_{\mathrm{c}}\right)$ is linearly related to $(\sin \theta / \lambda)^{2}$. By a least square treatment of data, $B$ was determined. From the Debye-Waller theory

$$
B=\left(\frac{8 \pi^{2}}{3}\right)\left\langle u^{2}\right\rangle,
$$

for a cubic crystal, where $\left\langle u^{2}\right\rangle$ is the mean-square amplitude of vibration. Further, $B$ may also be expressed as

$$
B=\left(\frac{6 h^{2}}{m k_{\mathrm{B}} T}\right) W(x),
$$

where $m$ is the mass, $T$ the absolute temperature and $h$ and $k_{\mathrm{B}}$ are the Planck and Boltzmann constants, respectively. The function $W(x)$ is given by

$$
W(x)\left[\frac{\phi(x)}{x^{2}}+\frac{1}{4 x}\right],
$$

where $\phi(x)$ is the Debye function and $x=\theta_{\mathrm{M}} / T, \theta_{\mathrm{M}}$ being the Debye temperature. Benson and Gill (1966) have tabulated values of $W(x)$ for a wide range of $x$ for small increments, from which $\theta_{\mathrm{M}}$ can be obtained from the value of $B$.

\subsection{Lattice strain and particle size determination}

When the size of the individual crystals is less than about $100 \mathrm{~nm}$, the term 'particle size' is usually used. When the crystallites of a material are smaller than $100 \mathrm{~nm}$, they have too small a number of parallel diffraction planes and so they produce broadened diffraction peaks instead of a sharp peak. Lattice strain present in the sample is another cause of broadening of Bragg diffraction peaks. In addition to this, there are instrumental factors such as unresolved $\alpha 1$ and $\alpha 2$ peaks, imperfect focusing which lead to line broadening.
There are various methods in practice to estimate the particle size. X-ray diffraction is a simpler and easier approach for the determination of precise particle size and lattice strain in powder samples. The principle involved in the X-ray diffraction approach is precise quantification of the broadening of the Bragg diffraction peaks. Scherrer equation, HallWilliamson method and Warren-Averbach method are some of the techniques based on this principle. Of the above methods, Scherrer equation method for the estimation of particle size does not take into account the broadening due to lattice strain present in the sample. As such in the present investigation, the lattice strains have been estimated using Hall-Williamson method. Recently, Rehani et al (2006) used this method to estimate the lattice strain and particle sizes of silver nanoparticles and composite silver nanoparticles. In this method, integral breadth of the diffraction peak is determined. The integral breadth is given by the integrated intensity divided by maximum intensity. Thus, the observed peak broadening, $B_{0}$, may be represented as

$$
B_{\mathrm{o}}=B_{\mathrm{i}}+B_{\mathrm{r}},
$$

where $B_{\mathrm{o}}$ is the observed peak broadening in radians, $B_{\mathrm{i}}$ the instrumental broadening in radians and $B_{\mathrm{r}}$ the broadening due to small particle size and lattice strain. The instrumental broadening has been estimated using a pure strainfree fine sodium chloride powder sample subjected to XRD under identical conditions as those for the strained metallic powders. Equation (9) holds good if the diffraction peaks exhibit purely Cauchy profile. However, when the diffraction peaks are partly Cauchy and partly Gaussian for profiles, the following relation among $B_{\mathrm{o}}, B_{\mathrm{i}}$ and $B_{\mathrm{r}}$ holds good (Rehani et al 2006).

$$
B_{\mathrm{r}}=\left[\left(B_{\mathrm{o}}-B_{\mathrm{i}}\right)\left(B_{\mathrm{o}}^{2}-B_{\mathrm{i}}^{2}\right)^{1 / 2}\right]^{1 / 2} .
$$

Now, according to Scherrer equation, the broadening due to small particle size may be expressed as

$$
B_{\mathrm{c}}=\frac{k \lambda}{t} \cos \theta,
$$

where $B_{\mathrm{c}}$ is the broadening solely due to small crystallite size, $K$ a constant whose value depends on particle shape and usually taken as unity, $t$ the crystallite size in nanometers, $\theta$ the Bragg angle and $\lambda$ the wavelength of incident X-ray beam in nanometers.

Similarly, according to Wilson (1949), broadening due to lattice strain may be expressed by the relation

$$
B_{\mathrm{s}}=\varepsilon \tan \theta,
$$

where $B_{\mathrm{S}}$ is the peak broadening due to lattice strain and $\varepsilon$ the strain distribution within the material and $\theta$ the Bragg angle. Based on (11) and (12), the total peak broadening, $B_{\mathrm{r}}$, may be expressed as

$$
B_{\mathrm{r}}=\frac{k \lambda}{t \cos \theta}+\varepsilon \tan \theta,
$$


which can be written as

$$
B_{\mathrm{r}} \cos \theta=\frac{k \lambda}{t}+\varepsilon \sin \theta \text {. }
$$

The plot of $B_{\mathrm{r}} \cos \theta / \lambda$ vs $\sin \theta / \lambda$ is a straight line with slope equal to $\varepsilon$ and hence the particle size ' $t$ ' can be estimated from the intercept. Typical Hall-Williamson plot between $B_{\mathrm{r}} \cos \theta / \lambda$ and $\sin \theta / \lambda$ is shown in figure 1 .

The lattice strains were determined from the plot of $B_{\mathrm{r}}$ $\cos \theta / \lambda$ against $\sin \theta / \lambda$, following standard procedures (Kaelble 1967). The measured half-widths were corrected for instrumental broadening with reference to a pure strain-free silicon powder. The variation of particle size with milling time is within the limits of experimental errors. This shows that while milling is enough to create strains, it affects the particle size to a measurable extent. A typical HallWilliamson plot is shown in figure 1 for $\mathrm{Pt}$ after grinding for $15 \mathrm{~h}$.

\section{Results and discussion}

The values of lattice strain, crystallite size, root mean square amplitude of vibrations, Debye-Waller factor and Debye temperature of Pt powders, ground for different durations, obtained in the present study, are given in table 1. As the objective of the present work is to investigate the strain dependence of Debye-Waller factors, the variation of lattice

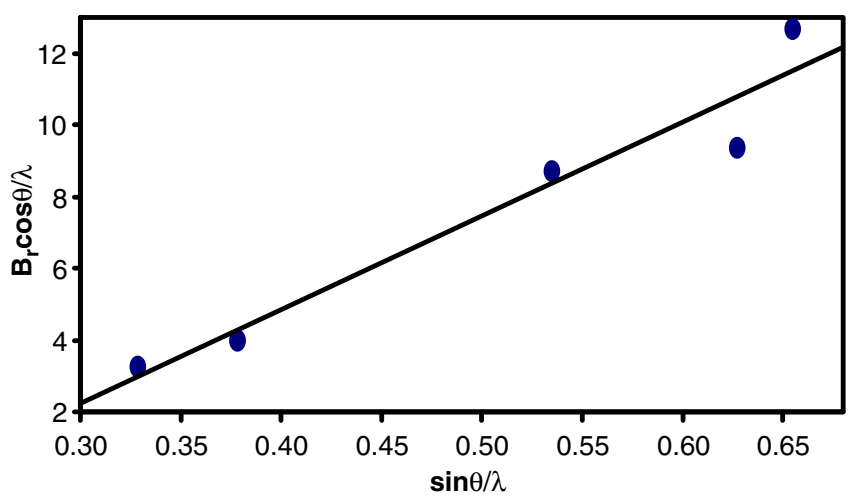

Figure 1. Plot of $B_{\mathrm{r}} \cos \theta / \lambda$ vs $\sin \theta / \lambda$ for Pt after milling for $15 \mathrm{~h}$. strain $(\varepsilon)$ and Debye-Waller factor $(B)$ for different milling times for $f c c$ metal $\mathrm{Pt}$ is shown in figure 2. Both lattice strain and Debye-Waller factor increase with milling time. This is similar to the observations of Inagaki et al (1971, 1973), Sirdeshmukh et al (1993) and Gopi Krishna and Sirdeshmukh (1993). The Debye-Waller factor increases with milling time and lattice strain in a slightly nonlinear fashion and crystallite size decreases with milling time. An extrapolation of the $B$ vs $\varepsilon$ curve to $\varepsilon=0$ gives the values of Debye-Waller factor, $0.28 \AA^{2}$. The zero-strain values of Debye-Waller factor is less than the values for the initial samples. The zero strain Debye-Waller factors of $0.28 \AA^{2}$ for $\mathrm{Pt}$ is close to the value of $0 \cdot 32 \AA^{2}$ obtained by Alexopoulos et al (1965) using powder X-ray diffraction. Thus, the Debye-Waller factors of Pt powder samples carry an effect due to lattice strain. While comparing the Debye-Waller factors calculated from the lattice dynamical models with experimental results, Vetelino et al (1972) attributed the difference to inaccuracies in the experimental values caused by neglecting TDS corrections. The repeated milling of the powder sample leads to lattice distortion which gives rise to microstrains in the lattice. These microstrains increase the contribution of static component of Debye-Waller factor. Thus, both lattice strain and the observed Debye-Waller factor, which is the sum of static and thermal components, increase with milling time. Thus, whenever Debye-Waller factors are determined from X-ray intensities on powder samples, it is desirable to make an estimate of the lattice strain and if the strain is large, a suitable correction is to be made as done in the present study. The Debye temperatures derived from the zero strain values of Debye-Waller factor of Pt is $244 \mathrm{~K}$. This value agree well with the values of $236 \mathrm{~K}$ (Alexopoulos et al $1965)$, obtained for $w$ from the powder X-ray diffraction.

Glyde (1967) derived the following relation between energy of vacancy formation $\left(E_{\mathrm{f}}\right)$ and the Debye temperature $(\theta)$ of a solid. The relation is

$$
E_{\mathrm{f}}=A(k / \hbar)^{2} M \theta^{2} a^{2},
$$

where $a$ is the interatomic spacing, $A$ a constant shown to be equal to $1.17 \times 10^{-2}, M$ the molecular weight and $\hbar$ and $k$ are the Plank's and Boltzmann's constants, respectively. Glyde recommended the use of X-ray based values in (15). The validity of (15) was verified for a number of $f c c, b c c$

Table 1. Values of lattice strain $(\varepsilon)$, crystallite size $(t)$, Debye-Waller factor $(B)$, root mean square amplitudes of vibration $\langle u\rangle$, Debye temperature $\left(\theta_{\mathrm{M}}\right)$ and energy of vacancy formation $\left(E_{\mathrm{f}}\right)$ of strained platinum nanopowders.

\begin{tabular}{lccccccc}
\hline Metal & Milling time & $\varepsilon \times 10^{3}$ & $t(\mathrm{~nm})$ & $\langle u\rangle(\AA)$ & $B\left(\AA^{2}\right)$ & $\theta_{\mathrm{M}}(\mathrm{K})$ & $E_{\mathrm{f}}(\mathrm{eV})$ \\
\hline $\mathrm{Pt}$ & 0 & 1.96 & 168 & $0 \cdot 1085(2)$ & $0.31(1)$ & $240(5)$ & 3.75 \\
& 5 & 2.08 & 126 & $0.1186(3)$ & $0.37(2)$ & $219(7)$ & $3 \cdot 12$ \\
& 10 & 2.55 & 81 & $0.1322(1)$ & $0.46(2)$ & $197(2)$ & $2 \cdot 53$ \\
& 15 & 2.96 & 48 & $0.1364(2)$ & $0.49(3)$ & $191(13)$ & $2 \cdot 37$ \\
& 20 & 3.52 & 34 & $0.1419(6)$ & $0.53(6)$ & $183(7)$ & $2 \cdot 18$ \\
& 25 & 4.21 & 26 & $0.1596(1)$ & $0.67(2)$ & $164(5)$ & 1.75 \\
\hline
\end{tabular}



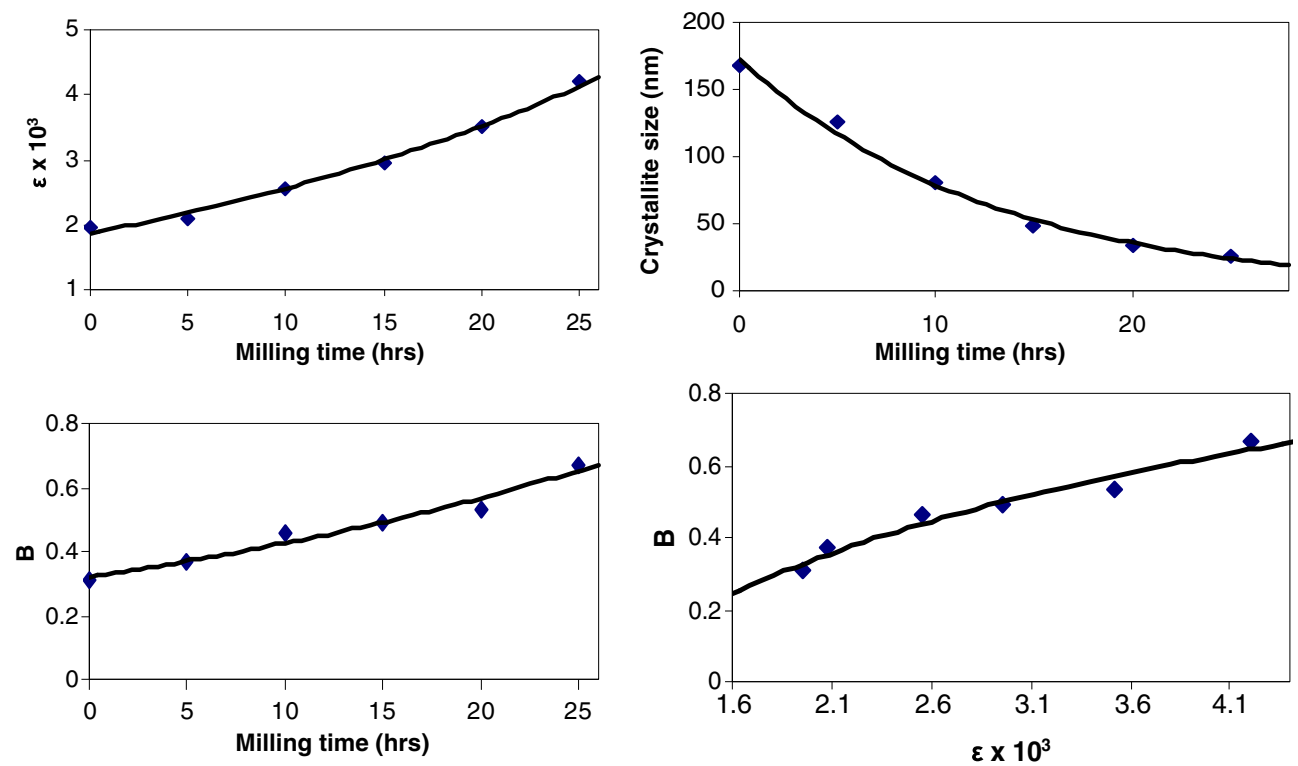

Figure 2. Milling time vs lattice strain, crystallite size and Debye-Waller factor and lattice strain vs Debye-Waller factor curves for Pt.

and $h c p$ metals (Springer Series in Material Science 2006). Therefore, X-ray Debye temperatures obtained in the present work have been used to study the variation of vacancy formation energy as a function of lattice strain in Pt. The values of vacancy formation energies are also included in table 1 .

\section{Conclusions}

Platinum powder was strained by milling for $25 \mathrm{~h}$. From a study of X-ray diffractograms recorded at different stages of milling, it is observed that milling for $25 \mathrm{~h}$ has systematic effect on the particle size. However, milling produces lattice strain and also enhances the effective Debye-Waller factor. By an extrapolation of the plot between the DebyeWaller factor and the lattice strain, zero strain Debye-Waller factors are obtained for Pt. The variation of energy of vacancy formation as a function of lattice strain has been studied.

\section{Acknowledgements}

The authors are grateful to the referee for suggestions. The authors thank the University Grants Commission, New Delhi, for financial assistance under special assistance programme (SAP) No. F.530/8/DRS/2009(SAP-I).

\section{References}

Alexopoulos K, Boskovits J, Mourikis S and Roilos M 1965 Acta Crystallogr. 19349

Benson G C and Gill E K 1966 Table of integral functions related to Debye-Waller factor (Ottawa: National Research Council of Canada)

Chipman D R and Paskin A 1959 J. Appl. Phys. 301938

Cromer D T and Waber J T 1965 Acta Crystallogr. 18104

Cromer D T and Liberman D 1970 J. Chem. Phys. 531891

Gopi Krishna N and Sirdeshmukh D B 1993 Indian J. Pure \& Appl. Phys. 31198

Glyde H R 1967 J. Phys. Chem. Solids (GB) 282061

Inagaki M et al 1971 J. Mater. Sci. 61520

Inagaki M, Furuhashi H, Ozeki T and Naka S 1973 J. Mater. Sci. 8312

International Tables for X-ray Crystallography 1968 (Birmingham: Kynoch Press) Vol. III

Kaelble E F 1967 Handbook of X-rays (New York: McGraw Hill)

Klug H P and Alexander L E 1974 X-ray diffraction procedures (USA: John Wiley and Sons)

Rehani B R, Joshi P B, Kirit N L and Pratap A 2006 Indian J. Pure \& Appl. Phys. 44157

Sirdeshmukh D B, Subhadra K G, Hussain K A, Gopi Krishna N and Raghavendra Rao B 1993 Cryst. Res. Technol. 2815

Springer Series in Material Science 2006 Micro- and macroproperties of solids

Vetelino J F, Gaur S P and Mitra S S 1972 Phys. Rev. B5 2360

Wilson A J C 1949 X-ray optics (London: Methuen) 